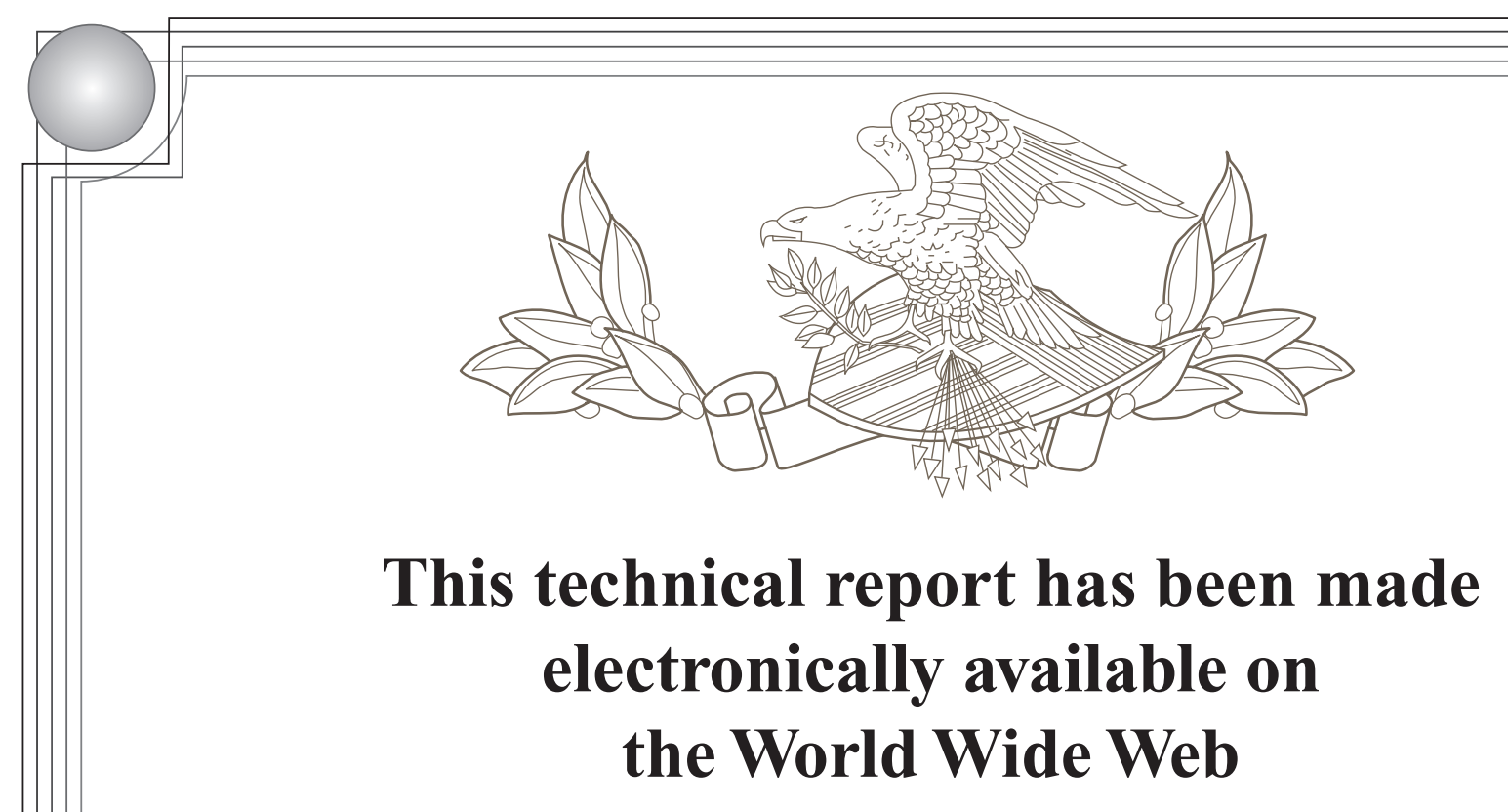

\title{
In memory of
}

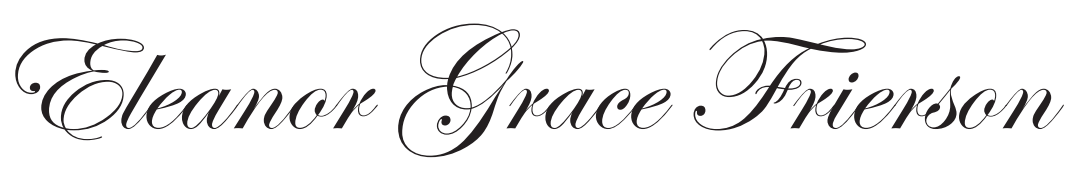

Deputy Director of the U.S. National Agricultural Library from 2000 until her retirement in December 2011, and a champion of global collaboration to improve public access

to U.S. federal and international science information;

Eleanor was honored as Federal Librarian of the Year in 2010, was a driving force behind the creation of Science.gov, serving as the first Co-Chair of the Science.gov Alliance and brought that same level of energy to the launch and growth of WorldWideScience.

Office of Scientific and Technical Information Office of Science U.S. Department of Energy May 2013 http://www.osti.gov/bridge/servlets/purl/4438096/4438096.pdf

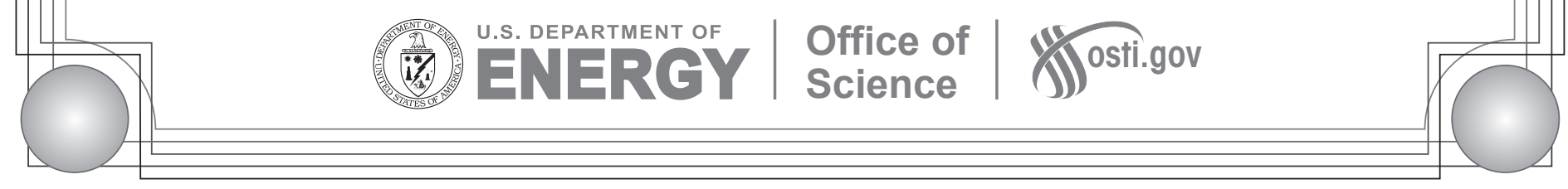


AECD - 2382

UNITED STATES ATOMIC ENERGY COMMISSION

ALPHA PARTICLES FROM FISSION AS RECORDED BY PHOTOGRAPHIC EMULSIONS

by

L. Marshall

Argonne National Laboratory

Date of Manuscript: $\quad$ July 8, 1948

Date Declassified: November 15, 1948

Issuance of this document does not constitute authority for declassification of classified copies of the same or similar content and title and by the same author.

Technical Information Branch, Oak Ridge, Tennessee

AEC, Oak Ridge, Tenn., 8-17-49--850-A1481

Printed in U.S.A.

PRICE 10 CENTS 


\section{DISCLAIMER}

This report was prepared as an account of work sponsored by an agency of the United States Government. Neither the United States Government nor any agency Thereof, nor any of their employees, makes any warranty, express or implied, or assumes any legal liability or responsibility for the accuracy, completeness, or usefulness of any information, apparatus, product, or process disclosed, or represents that its use would not infringe privately owned rights. Reference herein to any specific commercial product, process, or service by trade name, trademark, manufacturer, or otherwise does not necessarily constitute or imply its endorsement, recommendation, or favoring by the United States Government or any agency thereof. The views and opinions of authors expressed herein do not necessarily state or reflect those of the United States Government or any agency thereof. 


\section{DISCLAIMER}

Portions of this document may be illegible in electronic image products. Images are produced from the best available original document. 


\title{
ALPHA PARTICLES FROM FISSION AS RECORDED BY PHOTOGRAPHIC EMULSIONS
}

\author{
By L. Marshall
}

\begin{abstract}
Eighty long-range alpha particles coincident with $\mathrm{U}^{235}$ fission have been observed in photographic plates. There is a tendency for the alpha particles to emerge with greatest energy at an angle slightly greater than $\mathbf{9 0}$ degrees from the heavy fragment. In accordance with previous observers, it is found that most alpha particles emerge approximately perpendicular. It is also found that the majority form an angle slightly obtuse to the heavy fragment. The energy of the alpha particles is found to be derived largely from the kinetic energy of the fission fragments. The alpha emitting fissions have approximately the same mass ratio as do normal fissions.
\end{abstract}

\section{INTRODUCTION}

The alpha particles coincident with fission were first observed during the war by L. W. Alvarez (see first paragraph of reference 2). Independently the same particles were observed in photographic plates by Green and Livesey who reported them at the Physical Society Conference at Cambridge in July 1946, and by San-Tsiang, Zah-Wei, Chastel and Vigneron, ${ }^{1}$ who were apparently the first to publish it in the open literature.

At approximately the same time, Demers, ${ }^{3}$ knowing of Alvarez' discovery, published an analysis of fissions observed in photographic plates. In an examination of 1500 fissions he found 6 fissions emitting alpha particles, a frequency of 1 in 250 fissions. Using a special emulsion consisting of a thin layer of a uranium salt sandwiched between two layers of emulsion, he was able to observe a gap in the fission track corresponding to the point at which the fission occurred. From the coincidence of the gap with the point of emission of the alpha track he calculated that the alpha particle was emitted less than $2 \times 10^{-14}$ seconds after fission. The maximum energy observed for the alpha particles was about $19 \mathrm{Mev}$.

Farwell, Segre', and Wiegand, ${ }^{2}$ in a publication of work done during the war, give the frequency of alpha emission in U235 as 1 in 250, a figure based on good statistics. By bombarding a film of U235 in an ion chamber with neutrons they were able to measure the bias and absorption curves for the alpha particles so produced and thus to identify them positively. The resolving time of their electrical circuits allowed them to ascertain that the alpha coincided with fission within $5 \times 10^{-6}$ seconds. The maximum energy observed was about $16 \mathrm{Mev}$.

A study of alpha particles from fission observed in photographic plates was made by Wollan, Moak, and Sawyer ${ }^{4}$ on twenty individual events. These particles were found to emerge at approximately right angles to the fission tracks. From this fact the authors conclude that the alpha particle is released at the instant of fission and not from either fission fragment. The maximum energy observed was 22.1 Mev.

A more extensive investigation using photographic plates was made by Green and Livesey. ${ }^{5}$ In an examination of 25,000 fission tracks, they found 1 light particle emitted in every 80 fissions. Most of these were of short range and many emerge at angles less than $\mathbf{7 0}$ degrees to the fission track.

AECD - 2382 
In view of the criterion to be discussed herein it would seem probable that a large number of these light particles are protons of the emulsion knocked on by the fission fragments, even though such a possibility has apparently been discarded by the authors.

\section{EXPERIMENTAL DATA}

In the present research, an examination of approximately 18,500 fissions has been made in Eastman Kodak emulsion NTB. Among these, 80 fissions were found which emitted long-range alpha particles. Approximately 150 fissions were found from which emerged tracks of protons of the emulsion knocked on by the fission particles. A criterion has been found by which to distinguish the knock-on protons from the true long-range alpha particles emitted in fission. An analysis has been made of the energies and angles of emission of the observed alpha particles and of the total length and of the separate lengths of the fission fragments of the alpha emitting fissions. Comparison has been made with the corresponding quantities of normal fission.

Eastman Kodak Nuclear Research Emulsion NTB, 50 microns thick, was soaked in uranium acetate for 5 minutes and while wet, exposed for a few seconds to a flux of thermal neutrons from the Argonne heavy water pile. The emulsion was developed for 20 minutes in full strength D-19 and fixed. Control plates were made, both with uranium acetate, unexposed to neutrons, and without uranium acetate, exposed to neutrons

The plates were scanned at about $1000 \mathrm{X}$ magnification, using a microscope projection table. When a fission track coincident with a light fragment track was found, the image of the event was traced at $1950 \mathrm{X}$ magnification. The different parts of the track were brought successively into focus and the height of the objective above the emulsion at each significant point was noted. The thickness of the emulsion was measured by finding the heights of the objective when the top and bottom grains of the emulsion were in focus. This last data measures the amount by which the emulsion has shrunk during development.

From the tracing, and the data on the depth of the track at various places, the true geometry of the event as it occurred in the emulsion at its original thickness has been calculated. Examples of such tracings are reproduced]jin Figure 1.

\section{EXPERIMENTAL RESULTS}

Approximately 18,500 fissions were examined. Of these about 230 fission tracks had light particle tracks emerging from them. Since it is easy to confuse tracks due to knock-on protons with those due to alpha particles emitted at the instant of fission, some criterion is necessary by which this distinction can be made.

In order that the long-range alphas might be sorted out, the following method was used. From the range-energy relation as determined by Lattes, Fowler, and Cuer for protons in emulsion of the same high silver bromide content, the velocity of each light particle, assuming that it was a proton, was calculated for its measured range. Since the mass of the proton may be neglected in comparison with the mass of a fission fragment, the approximate relation is valid:

$$
\mathrm{V}_{\mathrm{p}}=2 \mathrm{v}_{\mathrm{F}} \cos \theta
$$

where $V_{p}$ and $V_{F}$ are the velocities of proton and of fission fragment, and where $\theta$ is the angle between their trajectories.

From this relation the velocity of the fission fragment was calculated and plotted against the range of the fission fragment measured from its end to the point where the light particle emerged at an acute angle. The range velocity plot of the points so obtained is given as Figure 2, except for a considerable number of points, corresponding to particles of long range, which lie off the top of the figure. A large fraction of the points below $18 \times 10^{8} \mathrm{~cm} / \mathrm{sec}$ cluster in a region compatible with a range velocity relation for fission fragments. 


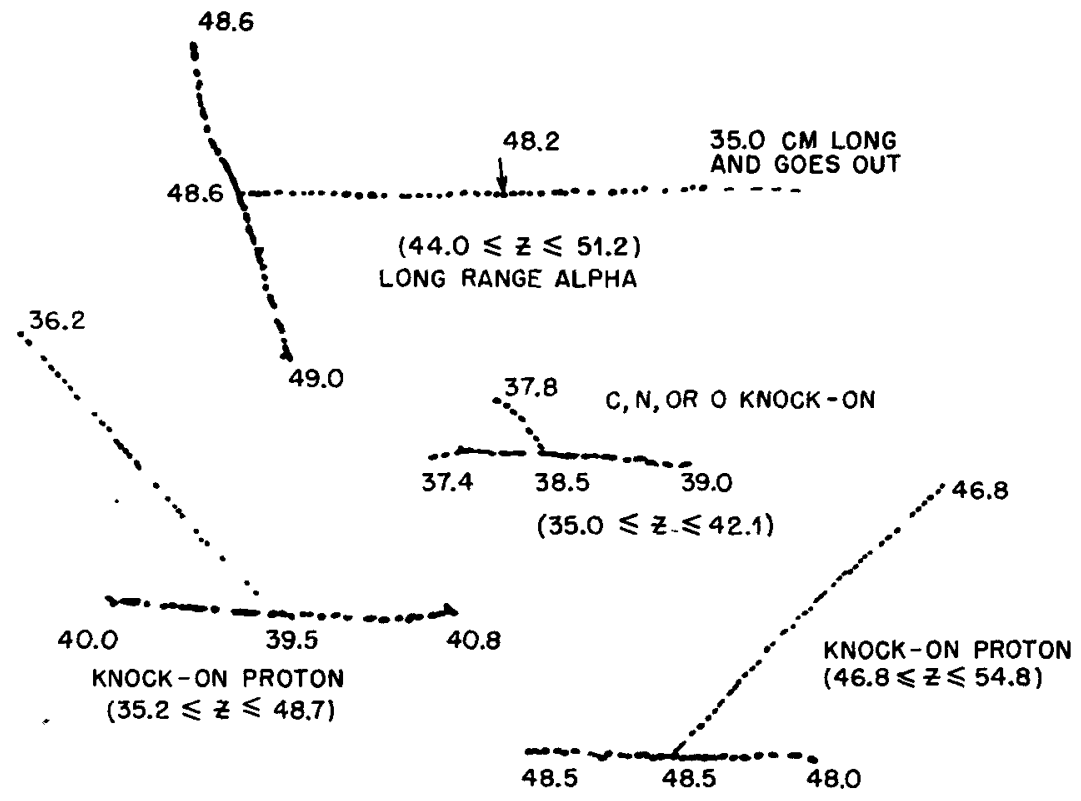

Figure 1. Examples of tracings. The numbers by the tracings give the $z$ coordinate in $10^{-4}$ inches.

The numbers in parentheses give the $z$ coordinates of the top and bottom of the emulsion in $10^{-4}$ inches. 


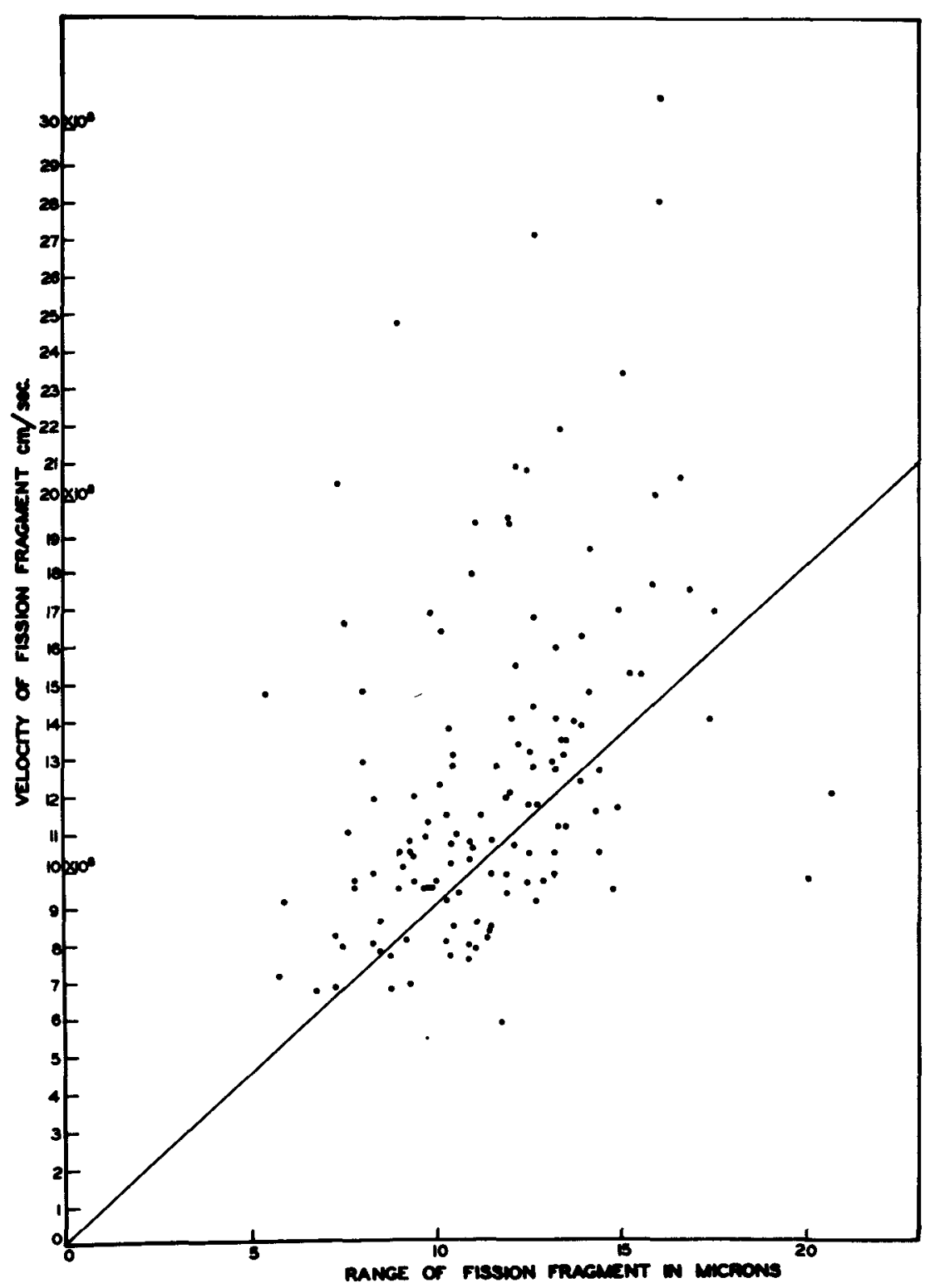

Figure 2. 
It has been found experimentally by various investigators $\mathrm{s}^{6,7,8}$ that the range of fission is proportional to the velocity, in accordance with the Bohr relation. The constant of proportionality can be calculated for the emulsion used here from the average length of normal fission in the emulsion together with the average masses and energies of the fission fragments. The average light fragment, with a mass of 93 and an energy of $96 \mathrm{Mev}$ has a velocity of $14 \times 10^{8} \mathrm{~cm} / \mathrm{sec}$. The heavy fragment of mass 141 and energy 64 has a velocity of $9.3 \times 10^{8} \mathrm{~cm} / \mathrm{sec}$. The average total range for normal fissions in this emulsion (see Figure 5) is 27.0 microns.

$$
\begin{aligned}
27.0 \text { microns } & =K(14+9.3) \times 10^{8} \mathrm{~cm} / \mathrm{sec} \\
K & =1.160 \times 10^{-8} \text { microns sec } / \mathrm{cm}
\end{aligned}
$$

It may be seen that this line lies well in the cluster of those points of Figure 2 which lie below $18 \times 10^{8}$ $\mathrm{cm} / \mathrm{sec}$.

The possibility that the light particle tracks were carbon, nitrogen, or oxygen recoils was also considered. In order to obtain the velocity of the particle from its range, assuming its mass to be about 14, the experimental range-velocity curves of Blackett and Lees ${ }^{9}$ for nitrogen and oxygen together with the theoretical extrapolations of Knipp and Teller ${ }^{10}$ have been used.

The relationship in equation 1 must now be modified by the reduced mass. It becomes

$$
\mathrm{V}_{\mathrm{N}}=2 \mathrm{~V}_{\mathrm{F}}\left\{\frac{\mathrm{M}_{\mathrm{F}}}{\mathrm{M}_{\mathrm{F}}+\mathrm{M}_{\mathrm{N}}}\right\} \cos \theta
$$

where $V_{N}$ and $M_{N}$ are velocity and mass of the nitrogen atom, and where $V_{F}$ and $M_{F}$ are velocity and mass of the fission fragment. The angle between the initial trajectory of the fission fragment and the trajectory of the nitrogen is $\theta$. Knowing $\mathbf{V}_{\mathbf{N}}$ and $\cos \theta$, one calculates $\mathrm{V}_{\mathbf{F}}$. Several of the shorter light particles when treated in this way as fragments with mass $\sim 14$, yielded satisfactory points on the range-velocity plot of Figure 2, and are plotted thereon.

The dozen farthest outlying points to the southeast and the northwest are probably accounted for by a few excessively large experimental errors. The maximum velocity on the average for a fission fragment; namely, that for a fragment of mass 93 and energy $94 \mathrm{Mev}$, is $13.9 \times 10^{8} \mathrm{~cm} / \mathrm{sec}$. This sets an average upper limit by which one can decide which points refer to proton recoils and which points refer to proper alpha particles. However, in applying this limit it must be remembered that the lack of resolution due to straggling in the nonhomogeneous emulsion and due to errors in measurement may allow points corresponding to true proton recoils to lie above this limit.

The light fragments corresponding to all points lying above $18 \times 10^{8} \mathrm{~cm} / \mathrm{sec}$ are considered to be true alpha particles. The fact that $90 \%$ of the points below $18 \times 10^{8} \mathrm{~cm} / \mathrm{sec}$ cluster in a region suitable to a range velocity curve for fission fragments, and that the remaining $10 \%$ lie around the edges of this region means that almost all of the corresponding light fragments are proton coils, and some few are recoils of carbon, nitrogen, and oxygen. Quite probably none of these points, even those which lie outside the cluster, correspond to alpha particles, since, if this were the case, one could expect an equivalent sprinkling of points in the regions away from the range-velocity region. Although it is possible that some few alpha particles are not counted by using this criterion, it is not believed that they can represent more than 10 to $15 \%$ at the most.

Using the criteria discussed for distinguishing the ture alpha particles from the knock-on protons and knock-on $\mathrm{C}, \mathrm{N}$, and $\mathrm{O}$ of the emulsion, there remain 80 long-range alpha particles from 18,500 fissions. This gives a frequency of 1 in 230 , a figure which agrees with the frequency noted by Farwell, et al. ${ }^{2}$ These are then the light particles corresponding to points above $18 \times 10^{8} \mathrm{~cm} / \mathrm{sec}$ in Figure 1 . Most of these points lie off the top of the graph by a large distance.

Out of 80,31 alpha tracks end in the emulsion. For the purposes of this analysis the remainder are not useless because they provide data on length of the total fission track, ratio of the lengths of 'the 2 fission fragments, and angle of emergence of the alpha particle.

In Figure 3 the energy of alpha particles is plotted vs. angle of emission showing both those alphas which leave the emulsion and those which stop in the emulsion. The angle of emission is the angle between the heavy fragment track and the alpha particle track. The energy of the alpha has been 


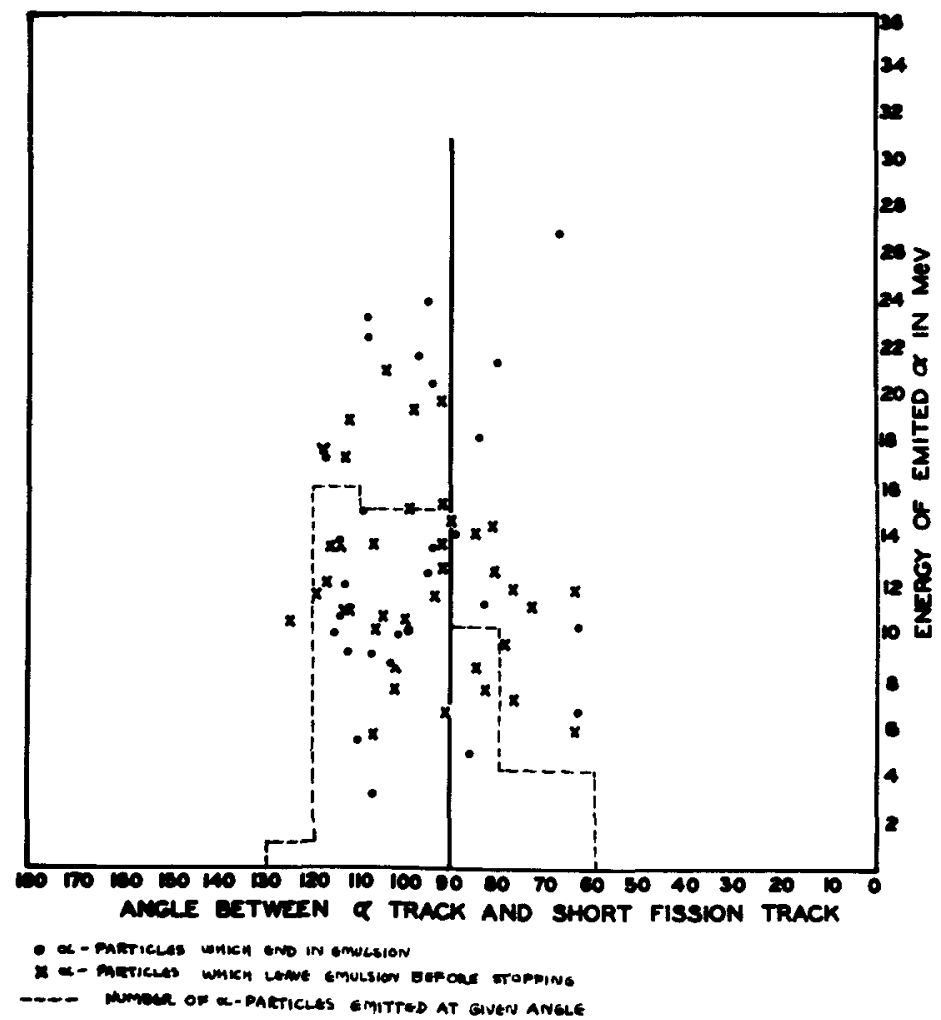

Figure 3.

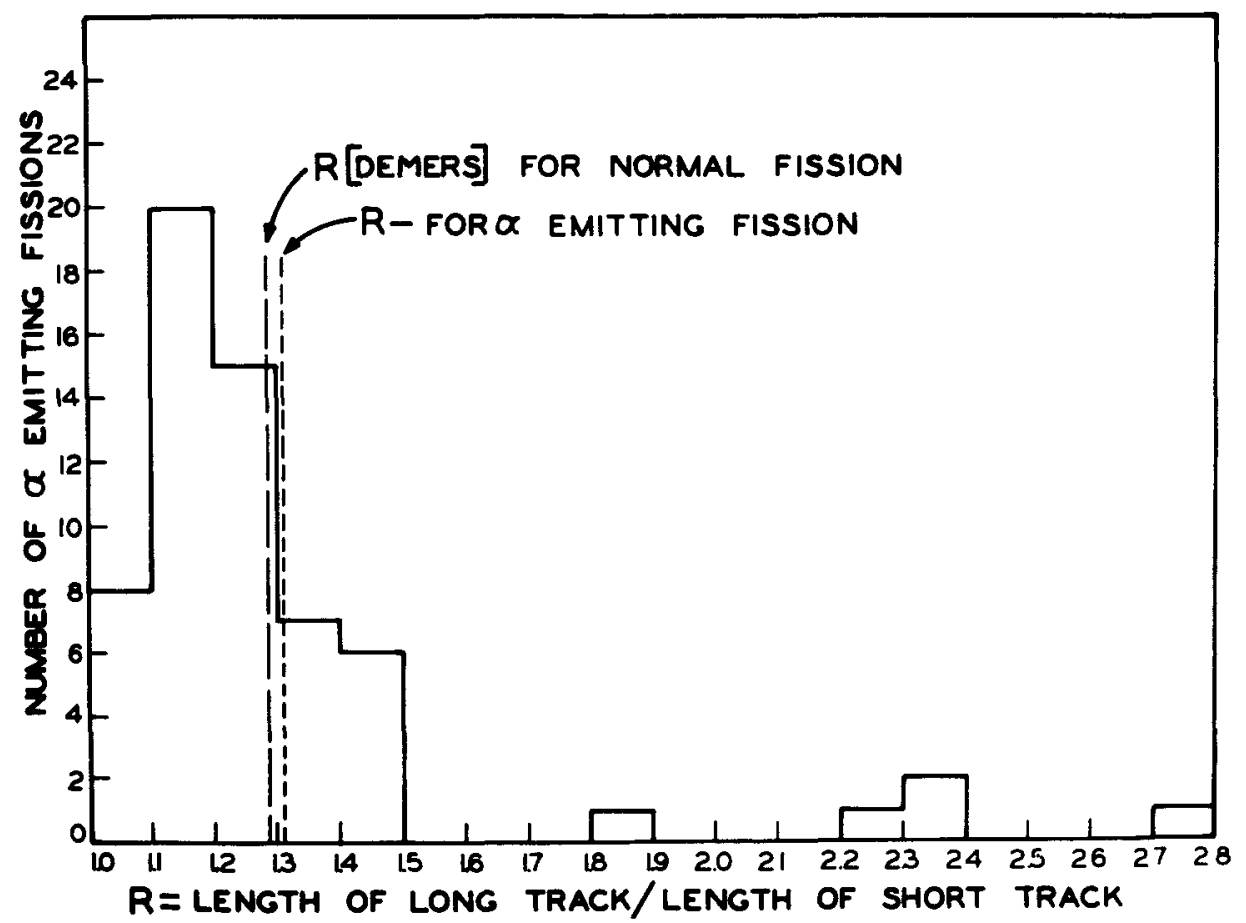

Figure 4. 
obtained from the range energy curves of Lattes, Fowler, and Cuer for alpha particles in high density silver bromide emulsion. There is a slight tendency for the more energetic alphas to be emitted at an angle obtuse to the heavier fragment, although the statistics are poor and the effect is probably within the statistical accuracy. Also the greater number of alphas are emitted at angles slightly obtuse to the heavy (short) fragment. Both effects, if real, are in agreement with the greater coulomb repulsion of the heavy fragment.

In Figure 4 is plotted the ratio of lengths of long to short fragment against the number of alpha emitting fissions showing that ratio. Within the experimental error the average ratio for alpha emitting fissions is equal to the average ratio observed by Demers for normal fissions. Even if those ratios greater than 1.7 are considered as anomalous errors and are omitted, the ratio is 1.24 , still equal to the ratio for normal fission well within the limits of error.

Since the ratio of the ranges of the fragments of a given fission is proportional ${ }^{11,12,13}$ to the ratio of their masses, in principle if straggling were small enough, Figure 4 could be interpreted as a chemical yield curve. The ordinate would then be proportional to per cent of fission into the mass ratio given by the abscissae, for alpha emitting fissions. Because the error due to straggling in the nonhomogeneous medium is so large, it is not possible to tell positively whether the width of the curve derives from a single type of fission or from all the types and frequencies of splitting observed for normal fission. Therefore, this curve unfortunately cannot be used as a chemical yield curve.

One may conclude, however, that alpha emitting fissions either have approximately the same chemical yield curve as normal fissions or else approximately correspond to those types of splitting most abundant among normal fissions.

There is no obvious correlation either of angle or of energy with length of either fission fragment or with total length.

According to Figure 5, the average total length of the alpha emitting fissions is about $94 \%$ of the average total length of normal fissions. This is equal to the decreased total range calculated, assuming a $16 \mathrm{Mev}$ alpha particle emitted in such a way that $8 \mathrm{Mev}$ subtracts from the kinetic energy of each fragment. Therefore, the energy of the alpha particles seems to be obtained at the expense of the kinetic energy of the fission fragments on the average.

From Figure 5 there can also be obtained some idea of the error due to straggling caused by inhomogeneity of the emulsion. It has been shown ${ }^{13}$ that the total range in air of the two fission fragments of normal fission has practically the same value for all mass ratios. However, one sees in Figure 5 that the lengths of normal fissions as measured in photographic emulsions range from less than 20 microns to more than 32 microns. This must be attributed to the fact that as the fission fragment goes through the emulsion it travels through regions of gelatine, and through erratically distributed grains of silver bromide in which it is slowed much faster than in the gelatine.

Figure 6 shows the actual energies observed for those alpha particles which stopped in the emulsion. In order to find frequency of emission as a function of energy these various energies must be weighted by the corresponding probability to leave the emulsion, shown as the dashed curve. There are few alpha particles emitted with low energy, the frequency of emission going through a maximum which is ill-defined due to the poor statistics of the observation.

In any given fission, one of the fission fragments is nearly always shorter than the other. In Figure 7, three curves are given: (1) the lengths of the short fission fragments are plotted against number, (2) the same is done for long fission fragments, and (3) finally a third curve gives the sum of the first two curves. These three curves all apply to alpha emitting fission only. One sees from Figure 7 that the curve for all the fission fragments from alpha emitting fission shows a single peak; that is, that the peaks of the curves for long and for short fragments are not markedly separated. This is in contrast to similar curves given by Demers ${ }^{3}$ for normal fission. The peaks of his curves for the long and for the short fragment are distinctly separated because they are much narrower (the average positions of the peaks have the same ratio as those for alpha emitting fission), and consequently the curve which is the sum of the first two shows two well-defined maxima.

However, Demers' curve for total length of the normal fission vs. number shows approximately the same spread as that observed here for normal fissions. Therefore, the widening of the rangenumber curves for long and for short fission fragments of alpha emitting fissions would not seem to 

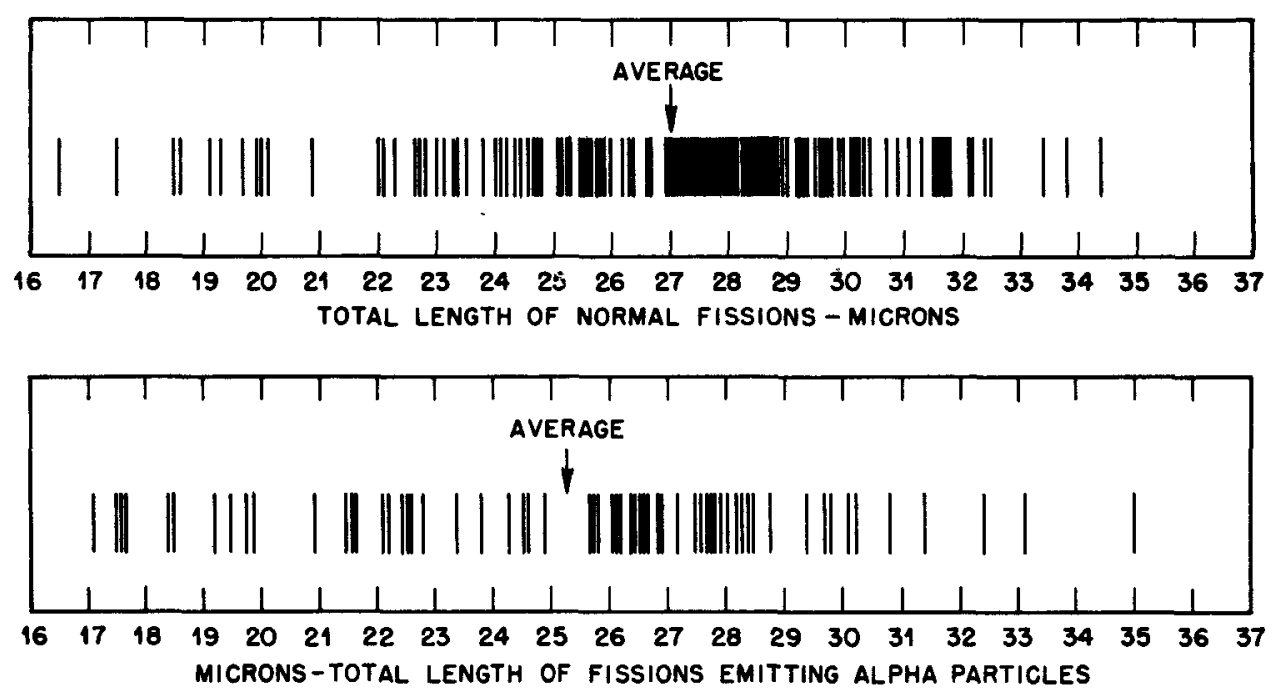

Figure 5,



Figure 6. 
be due to a less homogeneous emulsion but rather to the alpha emitting process itself. Probably it relates to the fact that the alpha particles come out with a wide range of energies. It may perhaps be repeated that the ratio of the average lengths of long and short fragments is not changed by alpha emission (although the average lengths themselves are decreased). This fact and the spread in alpha particle energies together with the apparent broadening of the range number curves of the fission fragments gives separate evidence that the energy for the alpha particle subtracts from the kinetic energy available to both fission fragments.

It should be pointed out that a curve, analogous to Figure 7, is given for alpha emitting fission by Green and Livesey. ${ }^{5}$ Their range number curve for all fission fragments together shows, however, two distinct peaks, apparently corresponding to the ranges of light and heavy fission fragments in emulsion. But the possibility exists that more than half of the light particles observed by them are proton recoils. Such an event does not divide the fission track into the lengths corresponding to the true ranges of the fission fragments. Consequently, their curve must be considered with this difficulty in mind.

In the same way in the collection of data for the present paper, the knock-on protons were at first confused with true alpha particles. The differences in grain densities of the two kinds of tracks are not marked, at least not with the methods of development used here, although such critical development methods can probably be found with a little care. In the course of plotting the data, it became obvious that a large number of the shorter light fragments were emitted at an angle acute to the heavy fragment, and also in such a way that the ratio of long to short fission fragment, measured from the origin of the light particle track, was unusually large. These facts are difficult to explain if the light fragments are alpha particles, but they are quite in keeping if the light fragments are knock-on protons. Consequently the criterion previously discussed was used to distinguish the knock-on light particles from the true long range alpha particles.

In Figure 8 are shown tracings of two events which on first sight appear to be the emission of two alpha particles at the instant of fission. However, of the two events, the shorter light fragment of one fits very well in length and direction the requirements for a knock-on proton. In the second case the shorter light fragment has the range and angle for a knock-on $\mathrm{C}, \mathrm{N}$, or $\mathrm{O}$ fragment. In both cases the long-light fragment is a true long-range alpha particle, and in both cases it emerges at slightly more than 90 degrees from the long (light) fission fragment. Two such events were found in 18,500 fissions. Also 80 long-range alpha events were found and about 150 knock-on protons, and knock on $C, N$. and $O$. The probability of the double events should be, therefore, of the order of $1 / 150 \times 1 / 80$ or 1 in 12,000 in agreement with what has been observed.

\section{CONCL USIONS}

The frequency of emission of long-range alpha particles in U235 fission was found here as 1 in 230 , in agreement with the previous investigators. ${ }^{2,3}$ The maximum energy of long-range alpha particle observed here is $26.4 \mathrm{Mev}$. There is a slight tendency for the alpha particles of highest energy to be emitted at an angle slightly greater than $\mathbf{9 0}$ degrees from the heavy fission fragment. Furthermore, the majority of alpha particles emerge at an angle slightly obtuse to the heavy fragment. The energy of the alpha particle is derived largely from the kinetic energy of the fission fragments. The magnitude of the ratio of average range of normal fission to average range of alpha-emitting fission checks well with that calculated, assuming a $16 \mathrm{Mev}$ alpha particle to be emitted in such a way that $8 \mathrm{Mev}$ subtracts from the kinetic energy of each fission fragment. The straggling of the fission fragments due to the nonhomogeneity of the emulsion is too great to allow the evaluation of a chemical yield curve for alpha emitting fissions. It can be concluded, however, that the alpha-emitting fissions have approximately the same mass ratio as do normal fissions. 
10]

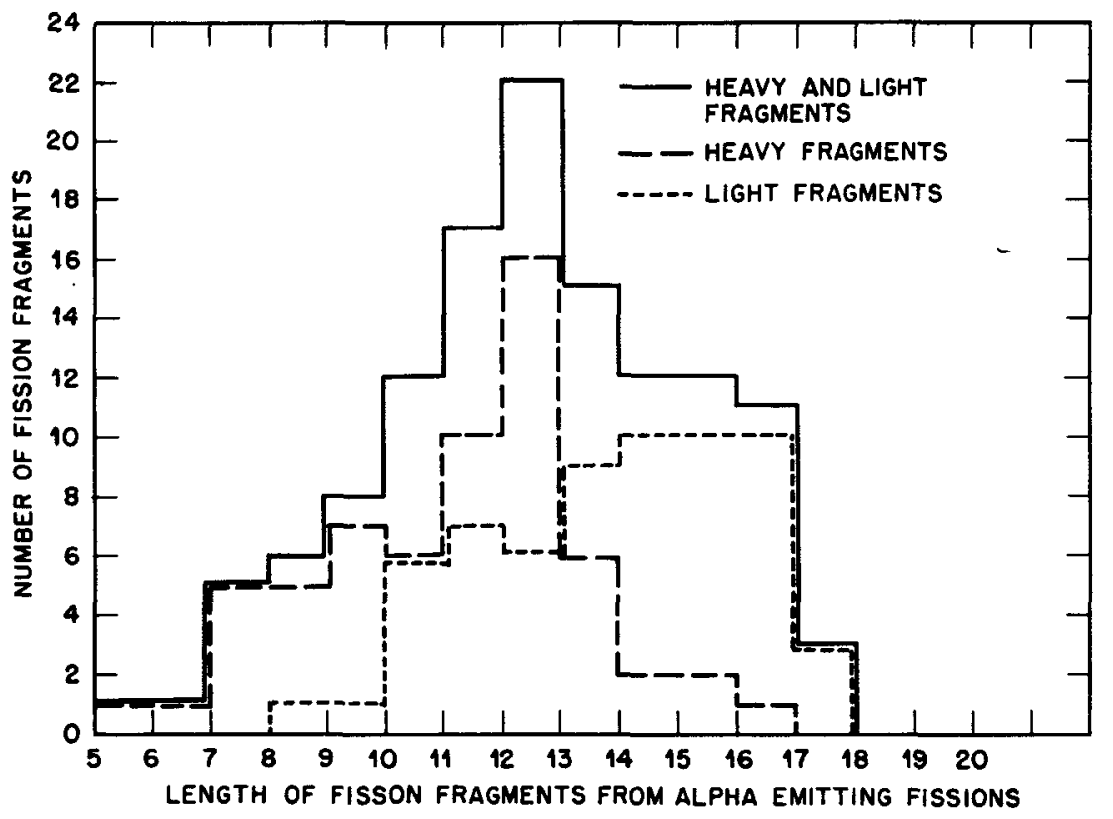

Figure 7.



Figure 8. 


\section{ACKNOWLEDGMENT}

Acknowledgment is made to Professor Enrico Ferml for valuable advice and discussions during the progress of this work.

\section{REFERENCES}

1. San-Tsiang, Zah-Wel, Chastel, and Vigneron, C.R. 223:986.

2. Farwell, Segré, and Wiegand, P.R. 71:327 (1947).

3. Pierre Demers, P.R. 70:974 (1946).

4. Wollan, Moak, and Sawyer, P.R. 72:447 (1947).

5. Green and Livesey, Nature 159:332 (1947).

6. Bröstrom, Böggild, and Lauritsen, P.R. 58:651 and 839 (1940); P.R. 59:275 (1941).

7. Bŏggild, Arröe, and sigurgiersson, P.R. 71:281 (1947).

8. Sherr and Peterson, Rev. Sci. Inst. 18:574 (1947).

9. Blackett and Lees, Proc. Roy. Soc. 134:658 (1932).

10. Knipp and Teller, P.R. 58:666 (1941).

11. Freedman, Metcalf, and Sugarman, Manhattan Project Report CC-1559 (4/8/44).

12. Finkle, Hoagland, Katcoff, and Sugarman, MPR CK-1806 (6/30/44); MPR CC-2076 (8/25/44).

13. Katcoff and Miskel, LAMS-441. 